\title{
Existence and stability of positive steady-state solutions for a Lotka-Volterra system with intraspecific competition
}

\author{
Meihua We ${ }^{i^{*}}$, Jinyong Chang ${ }^{2}$ and Lan $\mathrm{Qi}^{1}$
}

${ }^{*}$ Correspondence:

meihuaw@sohu.com

'Department of Mathematics and

Statistics, Yulin University, Yulin,

Shaanxi 719000, P.R. China

Full list of author information is

available at the end of the article

\begin{abstract}
In this paper, we investigate the existence and stability of the positive steady-state solutions for a Lotka-Volterra system with intraspecific competition by using the Lyapunov-Schmidt reduction technique. To do this, we must firstly obtain the semi-trivial steady states as their base, which extend the method used in the previous studies. Our results show that the two competition species with intraspecific competition can coexist for bigger regions of the diffusion rate $\mu$ and also complete the existing works.
\end{abstract}

MSC: $35 \mathrm{~K} 57$

Keywords: steady-state solutions; Lyapunov-Schmidt reduction technique; existence; stability

\section{Introduction}

The maintenance of biodiversity has received increasing attention from ecologists and mathematicians. Moreover, resource competition is thought as an important factor in driving evolutionary diversification, in which intraspecific competition for resources plays a major role; see [1-4]. The classical Lotka-Volterra competition system [5-8] gives a better description of the population competition, whose dynamical behaviors have been studied extensively; see [9-16] and the references therein. In this paper a competitive LotkaVolterra diffusion model of two slightly different species is studied, namely, the two species are identical except for their intraspecific competition rates. For this system, once the diffusion is involved biologically, it would be very interesting to find out whether and when the two species competing for the same limited resources to survive can coexist.

To begin with, we present the classical Lotka-Volterra system with spatially inhomogeneous terms as follows

$$
\begin{cases}u_{t}=\mu \Delta u+u[a(x)-u-v], & x \in \Omega, t>0, \\ v_{t}=\mu \Delta v+v[a(x)-u-v], & x \in \Omega, t>0, \\ \frac{\partial u}{\partial n}=\frac{\partial v}{\partial n}=0, & x \in \partial \Omega, t>0,\end{cases}
$$

where $\Omega$ is a bounded domain in $R^{N}$ with smooth boundary $\partial \Omega, \mu$ is the diffusion rate, $u(x, t)$ and $v(x, t)$ denote the densities of two competing species, and $a(x)$ represents the intrinsic growth rate of species.

O2014 Wei et al.; licensee Springer. This is an Open Access article distributed under the terms of the Creative Commons Attribution License (http://creativecommons.org/licenses/by/2.0), which permits unrestricted use, distribution, and reproduction in any medium, provided the original work is properly cited. 
The system above shows that the two species have the same diffusion rates and intrinsic growth rates. However, the two species with slight difference, such as the original species and the mutant, are common in biology; they correspond to the perturbed systems of (1.1), see [17-21] for example, and are originally introduced in [17]. The two species with different diffusion rates discussed in [17] correspond to the system

$$
\begin{cases}u_{t}=\mu \Delta u+u[a(x)-u-v], & x \in \Omega, t>0, \\ v_{t}=(\mu+\tau) \Delta v+v[a(x)-u-v], & x \in \Omega, t>0,\end{cases}
$$

where $\tau>0$. It is shown from $(u, v) \rightarrow(\theta, 0)(\theta$ given in the next section) as $t \rightarrow \infty$ that the slower diffuser can drive the faster one to extinction, and thus the two species cannot coexist. In [18], it is further stated that the slower diffuser may fail with a time periodic function $a(x, t)$ instead of $a(x)$. In [19], the model with small variations of intrinsic growth rates is given by

$$
\begin{cases}u_{t}=\mu \Delta u+u[a(x)+\tau g(x)-u-v], & x \in \Omega, t>0, \\ v_{t}=\mu \Delta v+v[a(x)-u-v], & x \in \Omega, t>0 .\end{cases}
$$

It is shown that the stability of the two competing species varies in a complicated way as $\mu$ increases, which implies that the two species only coexist for small regions of $\mu$ if they could. For $a(x)+\tau g(x)$ taking the form $a+\tau g(x)$ in [20], the authors further illustrate that the mutant can always survive and invade, but the original species can only coexist in some cases. Furthermore, in [21] the system with different interspecific competition rates is given as

$$
\begin{cases}u_{t}=\mu \Delta u+u[a(x)-u-(1+\tau g(x)) v], & x \in \Omega, t>0 \\ v_{t}=\mu \Delta v+v[a(x)-v-(1+\tau h(x)) u], & x \in \Omega, t>0\end{cases}
$$

Then a new structure of coexistence states is obtained and the two species can coexist even for bigger regions of $\mu$.

Motivated by the studies above, in this paper, we continue the analytic works for another perturbation system

$$
\begin{cases}u_{t}=\mu \Delta u+u[a(x)-(1+\tau g(x)) u-v], & x \in \Omega, t>0, \\ v_{t}=\mu \Delta v+v[a(x)-(1+\tau h(x)) v-u], & x \in \Omega, t>0, \\ \frac{\partial u}{\partial n}=\frac{\partial v}{\partial n}=0, & x \in \partial \Omega, t>0,\end{cases}
$$

where $\tau$ is a small positive constant (i.e., $\tau \ll 1)$ and $g(x), h(x)$ are smooth functions, which indicates that the two species only have different intraspecific competition rates. Our main purpose is to study the existence and stability of the positive steady-state solutions (that is, the coexistence states) of (1.2) by using the Lyapunov-Schmidt reduction technique, the implicit function theorem combined with finite covering theorem and the perturbation theory for completing the previous studies. To do this, however, we must firstly get the semi-trivial steady-state solutions of (1.2) according to [22], which is different from the corresponding results already known, and extend the method used in the existing works. The main results we obtained show that the two competition species with different intraspecific competition can also coexist for bigger regions of the diffusion rate $\mu$, but it 
is also important to caution that the conclusions for stability obtained in this paper are contrary to the ones analyzed in [21].

The rest of this paper is organized as follows. In Section 2, from [22] we firstly give the semi-trivial steady-state solutions of (1.2), and then analyze the stability of the solutions. In Section 3, on the basis of the semi-trivial solutions obtained in Section 2, we investigate the existence and nonexistence of positive steady-state solutions of (1.2) by using the Lyapunov-Schmidt reduction technique, the implicit function theorem and the finite covering theorem. In Section 4, combining stability theory with perturbation theory, we further discuss the stability of positive steady-state solutions in detail.

\section{Existence and stability of semi-trivial steady-state solutions}

In this section we firstly establish the existence of semi-trivial steady-state solutions of (1.2) by [22], whose proof is omitted. Then we simply analyze the stability of the semitrivial solutions.

Lemma 2.1 (see [22]) Suppose that $\lambda$ is a positive parameter and that either $\beta(x)>0$ on an open subset of $\partial \Omega$, or the boundary condition is a Dirichlet condition on part of $\partial \Omega$, or $\int_{\Omega} m(x) d x<0$ holds. The principal eigenvalue $\sigma_{1}$ of

$$
\begin{aligned}
& \nabla \cdot(d(x) \nabla \phi)+\lambda m(x) \phi=\sigma \phi, \quad x \in \Omega, \\
& d(x) \frac{\partial \phi}{\partial n}+\beta(x) \phi=0, \quad x \in \partial \Omega
\end{aligned}
$$

is positive if and only if $0<\lambda^{+}<\lambda$, where $\lambda^{+}$is the positive principal eigenvalue of

$$
\begin{aligned}
& \nabla \cdot(d(x) \nabla \phi)+\lambda m(x) \phi=0, \quad x \in \Omega, \\
& d(x) \frac{\partial \phi}{\partial n}+\beta(x) \phi=0, \quad x \in \partial \Omega .
\end{aligned}
$$

If $\beta(x) \equiv 0$ and $\int_{\Omega} m(x) d x>0$, then $\sigma_{1}>0$ for all $\lambda>0$.

Consider the following system:

$$
\begin{aligned}
& u_{t}=\nabla \cdot d(x) \nabla u+f(x, u), \quad x \in \Omega, t>0, \\
& d(x) \frac{\partial u}{\partial n}+\beta(x) u=0, \quad x \in \partial \Omega, t>0
\end{aligned}
$$

where $d(x) \in C^{1+\alpha}(\bar{\Omega}), d(x) \geq d_{0}>0, \beta(x) \in C^{1+\alpha}(\bar{\Omega}), \beta(x) \geq 0, f(x, u)$ is Lipschitz in $u$ and is a measurable function in $x$ which is bounded if $u$ is restricted to a bounded set and $f(x, 0)=0$.

Lemma 2.2 (see [22]) Suppose that $f(x, u)=q(x, u) u$ with $q(x, u)$ of class $C^{2}$ in $u$ and $C^{\alpha}$ in $x$, and there exists $K>0$ such that $q(x, u)<0$ for $u>K$. If the principal eigenvalue $\sigma_{1}$ is positive in the problem

$$
\begin{aligned}
& \nabla \cdot d(x) \nabla \psi+q(x, 0) \psi=\sigma \psi, \quad x \in \Omega, \\
& d(x) \frac{\partial \psi}{\partial n}+\beta(x) \psi=0, \quad x \in \partial \Omega,
\end{aligned}
$$


then (2.1) has a minimal positive equilibrium $u^{*}$, and all solutions to (2.1) that are initially positive on an open subset of $\Omega$ are eventually bounded by orbits which increase toward $u^{*}$ as $t \rightarrow \infty$.

Lemma 2.3 (see [22]) Suppose that the hypotheses of Lemma 2.2 are satisfied and that $f(x, u)=q(x, u) u$ with $q(x, u)$ strictly decreasing in $u$ for $u \geq 0$. Then the minimal positive equilibrium $u^{*}$ is the only positive equilibrium for (2.1).

For the further discussions, we make the following assumption on $a(x)$.

(H) $a(x)$ is Hölder continuous on $\bar{\Omega}$ and $\int_{\Omega} a(x) d x>0$.

Then, from Lemmas 2.1-2.3, it is well known that

$$
\mu \Delta \theta+\theta(a(x)-\theta)=0, \quad x \in \Omega, \quad \frac{\partial \theta}{\partial n}=0, \quad x \in \partial \Omega
$$

has only a positive solution $\theta(x, \mu)$ denoted by $\theta(\mu)$ when the condition $(\mathrm{H})$ is valid, and it is also easily found that

$$
\mu \Delta u+u[a(x)-(1+\tau g(x)) u]=0, \quad x \in \Omega, \quad \frac{\partial u}{\partial n}=0, \quad x \in \partial \Omega
$$

and

$$
\mu \Delta v+v[a(x)-(1+\tau h(x)) v]=0, \quad x \in \Omega, \quad \frac{\partial v}{\partial n}=0, \quad x \in \partial \Omega
$$

respectively have unique positive equilibriums $\tilde{u}(x, \mu, \tau)$ and $\tilde{v}(x, \mu, \tau)$, denoted by $\tilde{u}(\mu, \tau)$ and $\tilde{v}(\mu, \tau)$, for $(\mathrm{H})$ and $\tau \ll 1$. Hence, we can find the following result.

Theorem 2.1 Assume that $(\mathrm{H})$ holds and $\tau \ll 1$. Then (1.2) has semi-trivial steady-state solutions $(\tilde{u}(\mu, \tau), 0)$ and $(0, \tilde{v}(\mu, \tau))$.

To discuss the stability of semi-trivial steady-state solutions $(\tilde{u}(\mu, \tau), 0)$ and $(0, \tilde{v}(\mu, \tau))$, we need to consider the eigenvalue problem

$$
\begin{aligned}
& \mu \Delta \phi+[a(x)-2(1+\tau g(x)) u-v] \phi-u \psi=\lambda \phi, \quad x \in \Omega, \\
& \mu \Delta \psi+[a(x)-2(1+\tau h(x)) v-u] \psi-v \phi=\lambda \psi, \quad x \in \Omega, \\
& \frac{\partial \phi}{\partial n}=\frac{\partial \psi}{\partial n}=0, \quad x \in \partial \Omega
\end{aligned}
$$

corresponding to the solution $(u, v)$. Due to $[23,24]$, one can show that $(2.3)$ has a principal eigenvalue $\lambda(\mu, \tau)$, which is real, algebraically simple, and all other eigenvalues have their real parts less than $\lambda(\mu, \tau)$. Therefore, the stability of $(\tilde{u}(\mu, \tau), 0)$ is decided by the principal eigenvalue $\hat{\lambda}(\mu, \tau)$ of the problem

$$
\mu \Delta \psi+[a(x)-\tilde{u}] \psi=\lambda \psi, \quad x \in \Omega, \quad \frac{\partial \psi}{\partial n}=0, \quad x \in \partial \Omega .
$$

Similarly, the stability of $(0, \tilde{v}(\mu, \tau))$ is dependent on the principal eigenvalue $\tilde{\lambda}(\mu, \tau)$ of the problem

$$
\mu \Delta \phi+[a(x)-\tilde{v}] \phi=\lambda \phi, \quad x \in \Omega, \quad \frac{\partial \phi}{\partial n}=0, \quad x \in \partial \Omega .
$$


Theorem 2.2 Suppose that the hypotheses of Theorem 2.1 are valid. If $\hat{\lambda}(\mu, \tau)<0(\hat{\lambda}(\mu, \tau)>$ $0)$, then $(\tilde{u}(\mu, \tau), 0)$ is asymptotically stable (unstable). If $\tilde{\lambda}(\mu, \tau)<0(\tilde{\lambda}(\mu, \tau)>0)$, then $(0, \tilde{v}(\mu, \tau))$ is asymptotically stable (unstable).

Remark 2.1 In this section, we only give a simple conclusion for the stability of semitrivial steady-state solutions of (1.2), whose detailed proofs are analogous to Section 3 of [21].

\section{Existence and nonexistence of positive steady-state solutions}

The research on the steady-state solutions in a competition-diffusion system is always a hot issue. In this section we establish the existence and nonexistence of positive steady states of (1.2). For this purpose, we must make use of the semi-trivial solutions obtained in Section 2 for the following results.

To discuss the steady states of (1.2), we deal with the elliptic system corresponding to (1.2), which takes the form as follows

$$
\begin{cases}\mu \Delta u+u[a(x)-(1+\tau g(x)) u-v]=0, & x \in \Omega, \\ \mu \Delta v+v[a(x)-(1+\tau h(x)) v-u]=0, & x \in \Omega, \\ \frac{\partial u}{\partial n}=\frac{\partial v}{\partial n}=0, & x \in \partial \Omega\end{cases}
$$

For convenience of the following discussions, we denote

$$
\begin{aligned}
& G(\mu)=\int_{\Omega} g(x) \theta^{3}(x, \mu) d x, \\
& H(\mu)=\int_{\Omega} h(x) \theta^{3}(x, \mu) d x
\end{aligned}
$$

and give the Sobolev spaces

$$
\begin{aligned}
& X=\left\{(y, z) \in W^{2, p}(\Omega) \times W^{2, p}(\Omega): \frac{\partial y}{\partial n}=\frac{\partial z}{\partial n}=0, x \in \partial \Omega\right\}, \\
& X_{1}=\operatorname{span}\{(\theta,-\theta)\}, \\
& X_{2}=\left\{(y, z) \in X: \int_{\Omega}(y-z) \theta d x=0\right\}, \\
& Y=L^{p}(\Omega) \times L^{p}(\Omega),
\end{aligned}
$$

where $p>N$ such that $W^{2, p} \hookrightarrow C^{1}(\bar{\Omega})$.

According to (2.2), it is obvious that (3.1) has nontrivial nonnegative solutions $\Upsilon_{\mu}=$ $\{(s \theta(x, \mu),(1-s) \theta(x, \mu)): s \in[0,1]\}$ for $\tau=0$. Then for $\tau \ll 1$ we will look for the solutions of (3.1) near $\Upsilon_{\mu}$, namely, the steady states of (1.2).

Theorem 3.1 Suppose that $G(\mu)$ and $H(\mu)$ have no common roots, and set $\mu_{1}$ and $\mu_{2}$ are the consecutive and simple roots of $G(\mu) H(\mu)$.

(i) If $G(\mu) H(\mu)<0$ in $\left(\mu_{1}, \mu_{2}\right)$, then there exists $\delta>0$ such that for $\mu \in\left(\mu_{1}+\delta, \mu_{2}-\delta\right)$ such that (1.2) has no positive steady-state solutions other than semi-trivial ones for $\tau \ll 1$.

(ii) If $G(\mu) H(\mu)>0$ in $\left(\mu_{1}, \mu_{2}\right)$, then for any sufficiently small $\delta>0$ and $\tau \ll 1,(1.2)$ has the positive steady-state solutions $(u(\mu, \tau), v(\mu, \tau))$ satisfying $(\mu, u(\mu, \tau), v(\mu, \tau))$ in the 
neighborhood of $\left(\mu_{1}-\delta, \mu_{2}+\delta\right) \times \Upsilon_{\mu}$ besides semi-trivial ones. Here

$$
\begin{aligned}
& u(\mu, \tau)=s(\mu, \tau)\left[\theta(\mu)+\tilde{y}_{1}(\mu, \tau)\right], \\
& v(\mu, \tau)=[1-s(\mu, \tau)]\left[\theta(\mu)+\tilde{z}_{1}(\mu, \tau)\right]
\end{aligned}
$$

with $s(\mu, 0)=s(\mu):=\frac{H(\mu)}{G(\mu)+H(\mu)}, \tilde{y}_{1}(\mu, 0)=0$ and $\tilde{z}_{1}(\mu, 0)=0$. Moreover, the positive steadystate solution branches $(u(\mu, \tau), v(\mu, \tau))$ connect with the semi-trivial ones $(u(\mu(\tau), \tau)$, $v(\underline{\mu}(\tau), \tau))$ and $(u(\bar{\mu}(\tau), \tau), v(\bar{\mu}(\tau), \tau))$, and the smooth functions $\underline{\mu}(\tau)$ and $\bar{\mu}(\tau)$ are defined on $\{\tau: \tau \geq 0\}$ such that $\underline{\mu}(0)=\mu_{1}$ and $\bar{\mu}(0)=\mu_{2}$.

Proof For $\tau \ll 1$, our purpose is to find the positive solutions of (3.1) near $\Upsilon_{\mu}$. Following the Lyapunov-Schmidt reduction technique [25], we know that $X=X_{1} \oplus X_{2}$ on the basis of the spaces defined above. Then we can set

$$
(u, v-\theta)=s(\theta(\mu),-\theta(\mu))+(y, z)
$$

that is to say, the solution form we will look for is given by

$$
(u, v)=(s \theta(\mu),(1-s) \theta(\mu))+(y, z)
$$

where $s \in[0,1]$ and $(y, z) \in X_{2}$ near $(0,0)$.

Substituting the expression above into (3.1), we have

$$
F(y, z, \mu, \tau, s):=\left(\begin{array}{c}
\mu \Delta y+(a-\theta) y-s \theta(y+z)+f_{1}(y, z, \mu, \tau, s) \\
\mu \Delta z+(a-\theta) z-(1-s) \theta(y+z)+f_{2}(y, z, \mu, \tau, s)
\end{array}\right)=\left(\begin{array}{l}
0 \\
0
\end{array}\right),
$$

where

$$
\begin{aligned}
& f_{1}(y, z, \mu, \tau, s)=-y(y+z)-\tau g(s \theta+y)^{2}, \\
& f_{2}(y, z, \mu, \tau, s)=-z(y+z)-\tau h[(1-s) \theta+z]^{2},
\end{aligned}
$$

and the map $F: X_{2} \times\left(\mu_{1}-\delta_{1}, \mu_{2}+\delta_{1}\right) \times\left(-\delta_{1}, \delta_{1}\right) \times\left(-\delta_{1}, 1+\delta_{1}\right) \rightarrow Y$ defined above is a smooth function. Obviously, on the basis of the semi-trivial steady states obtained in Section 2, we have

$$
F(0,0, \mu, 0, s)=0, \quad F(0, \tilde{v}-\theta, \mu, \tau, 0)=0, \quad F(\tilde{u}-\theta, 0, \mu, \tau, 1)=0 .
$$

Let $L(\mu, s)=D_{(y, z)} F(0,0, \mu, 0, s) \in \mathcal{L}(X, Y)$. Then

$$
L(\mu, s)=\left(\begin{array}{cc}
\mu \Delta+a-\theta-s \theta & -s \theta \\
-(1-s) \theta & \mu \Delta+a-\theta-(1-s) \theta
\end{array}\right),
$$

which is denoted by $L$, and $L$ is a Fredholm operator of index zero since $X$ is compactly imbedded in $Y$. It is easy to check that $(\theta,-\theta)$ is in the kernel of $L$. Due to $\theta>0$, we know that 0 is a simple eigenvalue of $L$, which leads to

$$
\operatorname{ker}(L)=\operatorname{span}\{(\theta,-\theta)\}=X_{1} .
$$


Define the projection operator $P=P(\mu, s): Y \rightarrow X_{1}$ as

$$
P\left(\begin{array}{l}
y \\
z
\end{array}\right)=\frac{1}{\int_{\Omega} \theta^{2}(x, \mu) d x}\left[(1-s) \int_{\Omega} \theta y d x-s \int_{\Omega} \theta z d x\right]\left(\begin{array}{c}
\theta \\
-\theta
\end{array}\right) .
$$

It is easily found that

$$
R(P)=X_{1}, \quad P^{2}=P, \quad L P=P L=0,
$$

which results in

$$
R(L)=\left\{(y, z) \in Y:(1-s) \int_{\Omega} \theta y d x-s \int_{\Omega} \theta z d x=0\right\} .
$$

According to the Lyapunov-Schmidt reduction technique, the equivalent expression of (3.3) is

$$
\left\{\begin{array}{l}
P(\mu, s) F(y, z, \mu, \tau, s)=0, \\
{[I-P(\mu, s)] F(y, z, \mu, \tau, s)=0 .}
\end{array}\right.
$$

We know that $L$ is an isomorphic mapping from $X_{2}$ to $Y$, then, by applying the implicit theorem to the second equation of (3.7), it can be solved to get a unique solution $(y, z)=$ $(y(\mu, \tau, s), z(\mu, \tau, s))$ near $(0,0)$. Furthermore, combining the finite covering theorem, there exists $\delta_{2}>0$ such that

$$
(y(\mu, \tau, s), z(\mu, \tau, s)):\left(\mu_{1}-\delta_{2}, \mu_{2}+\delta_{2}\right) \times\left(-\delta_{2}, \delta_{2}\right) \times\left(-\delta_{2}, 1+\delta_{2}\right) \rightarrow X_{2}
$$

and

$$
y(\mu, 0, s)=0, \quad z(\mu, 0, s)=0 .
$$

Thus, the solvability of (3.3) is converted to that $(y(\mu, \tau, s), z(\mu, \tau, s))$ satisfies the first equation of (3.7), that is,

$$
\operatorname{PF}(y(\mu, \tau, s), z(\mu, \tau, s), \mu, \tau, s)=0 \text {. }
$$

Combining with (3.4), we have

$$
\begin{aligned}
& y(\mu, \tau, 0)=0, \quad z(\mu, \tau, 0)=\tilde{v}-\theta, \\
& y(\mu, \tau, 1)=\tilde{u}-\theta, \quad z(\mu, \tau, 1)=0 .
\end{aligned}
$$

On the basis of the definition of $P$, we can get a smooth function $\xi(\mu, \tau, s)$ satisfying

$$
\xi(\mu, \tau, s)\left(\begin{array}{c}
\theta \\
-\theta
\end{array}\right)=P(\mu, s) F(y(\mu, \tau, s), z(\mu, \tau, s), \mu, \tau, s) .
$$

Hence we need to solve $\xi(\mu, \tau, s)=0$. From (3.8) and (3.9), we can find

$$
\xi(\mu, 0, s)=\xi(\mu, \tau, 0)=\xi(\mu, \tau, 1) \equiv 0,
$$


and then there exists a smooth function $\xi_{1}(\mu, \tau, s)$ such that

$$
\xi(\mu, \tau, s)=\tau s(1-s) \xi_{1}(\mu, \tau, s) .
$$

Moreover, we know that the solutions $s=0$ and $s=1$ correspond to the semi-trivial solutions $(0, \tilde{v})$ and $(\tilde{u}, 0)$ of $(3.1)$, respectively. Consequently, it remains to solve $\xi_{1}(\mu, \tau, s)=0$.

From (3.11), it follows that

$$
\xi_{\tau}(\mu, 0, s)=s(1-s) \xi_{1}(\mu, 0, s)
$$

Differentiate (3.10) with respect to $\tau$, set $\tau=0$ and combine with (3.6) and (3.8), which leads to

$$
\begin{aligned}
\xi_{\tau}(\mu, 0, s)\left(\begin{array}{c}
\theta \\
-\theta
\end{array}\right) & =P(\mu, s) L(\mu, s)\left(\begin{array}{l}
y_{\tau}(\mu, 0, s) \\
z_{\tau}(\mu, 0, s)
\end{array}\right)+P(\mu, s) F_{\tau}(0,0, \mu, 0, s) \\
& =P(\mu, s) F_{\tau}(0,0, \mu, 0, s) .
\end{aligned}
$$

From (3.3), we obtain

$$
F_{\tau}(0,0, \mu, 0, s)=\left(\begin{array}{c}
-s^{2} g \theta^{2} \\
-(1-s)^{2} h \theta^{2}
\end{array}\right)
$$

and then it follows from (3.5) that

$$
P(\mu, s) F_{\tau}(0,0, \mu, 0, s)=s(1-s) \frac{(1-s) H(\mu)-s G(\mu)}{\int_{\Omega} \theta^{2}(x, \mu) d x}\left(\begin{array}{c}
\theta \\
-\theta
\end{array}\right) .
$$

Hence, according to (3.12)-(3.14), we have

$$
\xi_{1}(\mu, 0, s)=\frac{(1-s) H(\mu)-s G(\mu)}{\int_{\Omega} \theta^{2}(x, \mu) d x} .
$$

For $G(\mu) H(\mu)<0$ in $\left(\mu_{1}, \mu_{2}\right)$, for example, we let $G(\tilde{\mu}) H(\tilde{\mu})<0, \tilde{\mu} \in\left(\mu_{1}, \mu_{2}\right)$, and then we obtain $\xi_{1}(\tilde{\mu}, 0, s) \neq 0$ when $\tilde{\mu} \in\left(\mu_{1}, \mu_{2}\right)$ and $s \in[0,1]$. By using the finite covering theorem, there exists $\delta_{3}>0$ taken smaller if necessary such that $\xi_{1}(\mu, \tau, s)=0$ has no solution for $(\mu, \tau, s) \in\left(\mu_{1}+\delta_{3}, \mu_{2}-\delta_{3}\right) \times\left(-\delta_{3}, \delta_{3}\right) \times\left(-\delta_{3}, 1+\delta_{3}\right)$. This results in statement (i) of Theorem 3.1.

For $G(\mu) H(\mu)>0$ in $\left(\mu_{1}, \mu_{2}\right)$, we can see that $\xi_{1}(\tilde{\mu}, 0, s(\tilde{\mu}))=0$ with $s(\mu)=\frac{H(\mu)}{G(\mu)+H(\mu)}$ and $\xi_{1, s}(\tilde{\mu}, 0, s(\tilde{\mu}))=-\frac{G(\tilde{\mu})+H(\tilde{\mu})}{\int_{\Omega} \theta^{2}(x, \tilde{\mu}) d x} \neq 0$ for $\tilde{\mu} \in\left[\mu_{1}, \mu_{2}\right]$ and $s \in[0,1]$ since $G(\mu)$ and $H(\mu)$ have no common roots. Combining the implicit function theorem with the finite covering theorem, we have that for $\delta_{4}>0$ chosen yet smaller if necessary and $(\mu, \tau, s) \in$ $\left(\mu_{1}-\delta_{4}, \mu_{2}+\delta_{4}\right) \times\left(-\delta_{4}, \delta_{4}\right) \times\left(-\delta_{4}, 1+\delta_{4}\right)$, the equation $\xi_{1}(\mu, \tau, s)=0$ has only solution given by the smooth function $s=s(\mu, \tau)$ with $s(\mu, 0)=s(\mu)$, which shows that (3.3) has solutions $(y, z)=(y(\mu, \tau, s), z(\mu, \tau, s))$ with $s=s(\mu, \tau)$. Due to $y(\mu, \tau, 0)=0$ and $z(\mu, \tau, 1)=0$ given in (3.9), we see

$$
y(\mu, \tau, s)=s y_{1}(\mu, \tau, s), \quad z(\mu, \tau, s)=(1-s) z_{1}(\mu, \tau, s),
$$


and then denote $y_{1}(\mu, \tau, s(\mu, \tau))$ and $z_{1}(\mu, \tau, s(\mu, \tau))$ by $\tilde{y}_{1}(\mu, \tau)$ and $\tilde{z}_{1}(\mu, \tau)$, respectively. From (3.8), it follows that $\tilde{y}_{1}(\mu, 0)=0$ and $\tilde{z}_{1}(\mu, 0)=0$. Therefore, for $(\mu, \tau, s) \in\left(\mu_{1}-\right.$ $\left.\delta_{4}, \mu_{2}+\delta_{4}\right) \times\left(-\delta_{4}, \delta_{4}\right) \times\left(-\delta_{4}, 1+\delta_{4}\right)$, the equation $\xi(\mu, \tau, s)=0$ has solutions given by $s=0, s=1$ and $s=s(\mu, \tau)$, which shows that (1.2) has not only semi-trivial steady-state solution branches, but also positive branches given by (3.2) which meet the semi-trivial ones whose form is discussed below.

Clearly, $s(\mu, \tau)=0$ and $s(\mu, \tau)=1$ correspond to the semi-trivial stationary solution of (1.2). For $\mu=\mu_{1}$, there is either $G\left(\mu_{1}\right)=0$ or $H\left(\mu_{1}\right)=0$ due to that $G(\mu)$ and $H(\mu)$ have no common roots. Without loss of generality, we take $G\left(\mu_{1}\right)=0$, and then $1-s\left(\mu_{1}, 0\right)=$ $1-s\left(\mu_{1}\right)=0$. Moreover, $\left.[1-s(\mu, \tau)]_{\mu}\right|_{(\mu, \tau)=\left(\mu_{1}, 0\right)}=\left.[1-s(\mu, 0)]_{\mu}\right|_{\mu=\mu_{1}}=\frac{G^{\prime}\left(\mu_{1}\right)}{H\left(\mu_{1}\right)} \neq 0$ since $\mu_{1}$ is the simple root of $G(\mu) H(\mu)$. Thus, by the implicit function theorem, we can obtain a unique solution of $1-s(\mu, \tau)=0$ defined by a smooth function $\mu=\underline{\mu}(\tau)$ for $\tau \in\left[0, \delta_{5}\right)$ with $\delta_{5}>0$ small enough and $\underline{\mu}(0)=\mu_{1}$, which implies that the corresponding semi-trivial steady-state solution of $(1.2)$ can be described by $(u(\underline{\mu}(\tau), \tau), v(\underline{\mu}(\tau), \tau))=$ $(\tilde{u}(\mu(\tau), \tau), 0)$. Similarly, for the case $H\left(\mu_{1}\right)=0$, we can get only the solution of $s(\mu, \tau)=$ 0 still given by $\mu=\mu(\tau)$, which corresponds to the semi-trivial steady-state solution $(u(\underline{\mu}(\tau), \tau), v(\underline{\mu}(\tau), \tau))=(0, \tilde{v}(\underline{\mu}(\tau), \tau))$ of $(1.2)$. Moreover, for $\mu=\mu_{2}$, similar results hold true, and the unique solution of $1-s(\mu, \tau)=0$ or $s(\mu, \tau)=0$ is denoted by $\mu=\bar{\mu}(\tau)$ with $\bar{\mu}(0)=\mu_{2}$. Thus the relevant solution of $(1.2)$ is given by $(u(\bar{\mu}(\tau), \tau), v(\bar{\mu}(\tau), \tau))=$ $(\tilde{u}(\bar{\mu}(\tau), \tau), 0)$ or $(u(\bar{\mu}(\tau), \tau), v(\bar{\mu}(\tau), \tau))=(0, \tilde{v}(\bar{\mu}(\tau), \tau))$.

Remark 3.1 The proof of Theorem 3.1 shows the existence of loops or branches of positive steady states just as [21], whose details are omitted here.

\section{Stability of positive steady-state solutions}

In this section, we analyze the stability of positive steady states $(u(\mu, \tau), v(\mu, \tau))$ of $(1.2)$. For $\tau=0$, we know $(u(\mu, 0), v(\mu, 0))=(s \theta(\mu),(1-s) \theta(\mu))$, and then the principal eigenvalue of the corresponding eigenvalue problem (2.3) is $\lambda=0$ because of $\theta(\mu)>0$, which shows that all other eigenvalues have negative real parts. Furthermore, for $\tau \ll 1$, since all other eigenvalues also have negative real parts by the perturbation theory [26], the stability of $(u(\mu, \tau), v(\mu, \tau))$ is determined by the principal eigenvalue $\lambda_{1}(\mu, \tau)$ near 0 of $(2.3)$, that is,

$$
\begin{aligned}
& \mu \Delta \phi+[a(x)-2(1+\tau g(x)) u-v] \phi-u \psi=\lambda_{1}(\mu, \tau) \phi, \quad x \in \Omega, \\
& \mu \Delta \psi+[a(x)-2(1+\tau h(x)) v-u] \psi-v \phi=\lambda_{1}(\mu, \tau) \psi, \quad x \in \Omega, \\
& \frac{\partial \phi}{\partial n}=\frac{\partial \psi}{\partial n}=0, \quad x \in \partial \Omega .
\end{aligned}
$$

Firstly, for $\tau \ll 1$, we can set

$$
\phi(\mu, \tau)=\theta(\mu)+\tau \phi_{1}(\mu, \tau), \quad \psi(\mu, \tau)=-\theta(\mu)+\tau \psi_{1}(\mu, \tau)
$$

in (4.1) with smooth functions $\phi_{1}(\mu, \tau)$ and $\psi_{1}(\mu, \tau)$. Furthermore, the emphasis is on the sign of $\lambda_{1}(\mu, \tau)$. Hence, for small $\tau$, we divide the discussions of the sign of $\lambda_{1}(\mu, \tau)$ into three cases, that is, $\mu$ close to $\mu_{1}, \mu$ close to $\mu_{2}$ and $\mu$ bounded away from $\mu_{1}$ and $\mu_{2}$. To do this, we must first prove the following lemmas. 
Lemma 4.1 For $\tau \ll 1$, the principal eigenvalue $\lambda_{1}(\mu, \tau)$ of (4.1) satisfies

$$
\frac{\lambda_{1}(\mu, \tau)}{\tau} \int_{\Omega}(\psi u-\phi v) d x=\int_{\Omega}\left[2 g \phi u v-2 h \psi u v+g \psi u^{2}-h \phi v^{2}\right] d x .
$$

Proof Multiply the first equation of (4.1) by $v$ and integrate over $\Omega$ to get

$$
\int_{\Omega} \phi v[\tau h v-u-2 \tau g u]-\psi u v d x=\lambda_{1}(\mu, \tau) \int_{\Omega} \phi v d x .
$$

In the same way, multiplying the second equation of (4.1) by $u$ and integrating by parts, we can obtain

$$
\int_{\Omega} \psi u[\tau g u-v-2 \tau h v]-\phi u v d x=\lambda_{1}(\mu, \tau) \int_{\Omega} \psi u d x .
$$

From (4.4) and (4.5), it suffices to show that (4.3) is valid.

Furthermore, on the basis of Lemma 4.1, we can discuss the sign of $\lambda_{1}(\mu, \tau)$.

Lemma 4.2 For $\mu \in\left[\mu_{1}+\epsilon, \mu_{2}-\epsilon\right], \epsilon>0$, we have the following conclusion:

$$
\lim _{\tau \rightarrow 0^{+}} \frac{\lambda_{1}(\mu, \tau)}{\tau}=-\frac{G(\mu) H(\mu)}{G(\mu)+H(\mu)} \frac{1}{\int_{\Omega} \theta^{2}(x, \mu) d x} .
$$

Proof It is obvious that $(u(\mu, \tau), v(\mu, \tau)) \rightarrow(s(\mu) \theta(\mu),[1-s(\mu)] \theta(\mu))$ and $(\phi(\mu, \tau), \psi(\mu$, $\tau)) \rightarrow(\theta(\mu),-\theta(\mu))$ as $\tau \rightarrow 0^{+}$from (3.2) and (4.2). Then, for $\tau \rightarrow 0^{+}$, it follows that

$$
\begin{aligned}
& \int_{\Omega}(\psi u-\phi v) d x \rightarrow-\int_{\Omega} \theta^{2}(x, \mu) d x, \\
& \int_{\Omega}\left[2 g \phi u v-2 h \psi u v+g \psi u^{2}-h \phi v^{2}\right] d x \\
& \quad \rightarrow 2 s(\mu)[1-s(\mu)] G(\mu)+2 s(\mu)[1-s(\mu)] H(\mu)-s^{2}(\mu) G(\mu) \\
& \quad-[1-s(\mu)]^{2} H(\mu) \\
& =\frac{G(\mu) H(\mu)}{G(\mu)+H(\mu)} .
\end{aligned}
$$

Thus, we can obtain (4.6) from (4.3), (4.7) and (4.8).

For the discussions below, without loss of generality, we can assume the case $G\left(\mu_{1}\right)=$

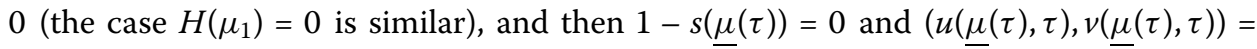
$(\tilde{u}(\mu(\tau), \tau), 0)$.

Lemma 4.3 Assume that $G\left(\mu_{1}\right)=0$. Then we have the following conclusion:

$$
\lim _{(\mu, \tau) \rightarrow\left(\mu_{1}, 0^{+}\right)} \frac{\lambda_{1}(\mu, \tau)}{\tau(\mu-\underline{\mu}(\tau))}=-\frac{G^{\prime}\left(\mu_{1}\right)}{\int_{\Omega} \theta^{2}\left(x, \mu_{1}\right) d x} .
$$

Proof According to Theorem 3.1, we know that $(\underline{\mu}(\tau), u(\underline{\mu}(\tau), \tau), v(\underline{\mu}(\tau), \tau))=(\underline{\mu}(\tau)$, $\tilde{u}(\underline{\mu}(\tau), \tau), 0)$ is the bifurcation point of the positive solutions to $(1.2)$. Then $\lambda_{1}(\underline{\mu}(\tau), \bar{\tau})=0$ 
and the eigenfunction $\psi(\underline{\mu}(\tau), \tau)$ satisfies

$$
\underline{\mu} \Delta \psi+[a(x)-\tilde{u}(\underline{\mu}(\tau), \tau)] \psi=0, \quad x \in \Omega, \quad \frac{\partial \psi}{\partial n}=0, \quad x \in \partial \Omega .
$$

Multiplying the equation above by $\tilde{u}(\underline{\mu}(\tau), \tau)$ and integrating by parts, we can get

$$
\int_{\Omega} g \psi(\underline{\mu}(\tau), \tau) \tilde{u}^{2}(\underline{\mu}(\tau), \tau) d x=0
$$

and then combining with $v(\mu(\tau), \tau)=0$ it follows that $\chi(\mu(\tau), \tau)=0$, where

$$
\chi(\mu, \tau):=\int_{\Omega}\left[2 g \phi u v-2 h \psi u v+g \psi u^{2}-h \phi v^{2}\right] d x
$$

in (4.3). Thus it leads to

$$
\chi(\mu, \tau)=\chi_{\mu}(\tilde{\mu}(\tau), \tau)(\mu-\underline{\mu}(\tau))
$$

by the mean value theorem for $\tilde{\mu}$ between $\mu$ and $\mu(\tau)$.

For $G\left(\mu_{1}\right)=0$, we know that $(u(\mu, \tau), v(\mu, \tau)) \rightarrow\left(\theta\left(\mu_{1}\right), 0\right),(\phi(\mu, \tau), \psi(\mu, \tau)) \rightarrow\left(\theta\left(\mu_{1}\right)\right.$, $\left.-\theta\left(\mu_{1}\right)\right),\left(u_{\mu}(\mu, \tau), v_{\mu}(\mu, \tau)\right) \rightarrow\left(s_{\mu}\left(\mu_{1}\right) \theta\left(\mu_{1}\right)+\theta_{\mu}\left(\mu_{1}\right),-s_{\mu}\left(\mu_{1}\right) \theta\left(\mu_{1}\right)\right),\left(\phi_{\mu}(\mu, \tau), \psi_{\mu}(\mu, \tau)\right) \rightarrow$ $\left(\theta_{\mu}\left(\mu_{1}\right),-\theta_{\mu}\left(\mu_{1}\right)\right)$ as $\tau \rightarrow 0^{+}$and $\mu \rightarrow \mu_{1}$. Then, differentiating (4.10) with respect to $\mu$, we obtain

$$
\begin{aligned}
\chi_{\mu}(\mu, \tau)= & \int_{\Omega}\left[2 g\left(\phi_{\mu} u v+\phi u_{\mu} v+\phi u v_{\mu}\right)-2 h\left(\psi_{\mu} u v+\psi u_{\mu} v+\psi u v_{\mu}\right)\right. \\
& \left.+g\left(\psi_{\mu} u^{2}+2 \psi u u_{\mu}\right)-h\left(\phi_{\mu} v^{2}+2 \phi v v_{\mu}\right)\right] d x \\
\rightarrow & -2 s_{\mu}\left(\mu_{1}\right) H\left(\mu_{1}\right)-G^{\prime}\left(\mu_{1}\right) \\
= & 2 \frac{G^{\prime}\left(\mu_{1}\right)}{H\left(\mu_{1}\right)} H\left(\mu_{1}\right)-G^{\prime}\left(\mu_{1}\right) \\
= & G^{\prime}\left(\mu_{1}\right)
\end{aligned}
$$

as $\tau \rightarrow 0^{+}$and $\mu \rightarrow \mu_{1}$. From (4.3), (4.11) and (4.12), we can get the relation (4.9).

In the same way, for $\mu$ close to $\mu_{2}$, the sign of $\lambda_{1}(\mu, \tau)$ is decided by the result below, whose details are omitted here.

Lemma 4.4 Assume that $G\left(\mu_{2}\right)=0$. Then we have the following conclusion:

$$
\lim _{(\mu, \tau) \rightarrow\left(\mu_{2}, 0^{+}\right)} \frac{\lambda_{1}(\mu, \tau)}{\tau(\mu-\bar{\mu}(\tau))}=-\frac{G^{\prime}\left(\mu_{2}\right)}{\int_{\Omega} \theta^{2}\left(x, \mu_{2}\right) d x} .
$$

Finally, based on Lemmas 4.2-4.4, the main result of this section is presented as follows.

Theorem 4.1 Suppose that $G(\mu)$ and $H(\mu)$ have no common roots, $\mu_{1}<\mu_{2}$ are two consecutive and simple roots of $G(\mu) H(\mu)$ and $G(\mu) H(\mu)>0$ in $\left(\mu_{1}, \mu_{2}\right)$. Then, for $\tau \ll 1$ and $\mu \in(\underline{\mu}(\tau), \bar{\mu}(\tau))$, we have: 
(i) If $G(\mu)>0$ and $H(\mu)>0$ in $\left(\mu_{1}, \mu_{2}\right)$, then the positive steady states $(u(\mu, \tau), v(\mu, \tau))$

of (1.2) are asymptotically stable;

(ii) If $G(\mu)<0$ and $H(\mu)<0$ in $\left(\mu_{1}, \mu_{2}\right)$, then $(u(\mu, \tau), v(\mu, \tau))$ is unstable.

Proof Now, we only consider the statement (i) (the statement (ii) can be proved similarly). The key point in the proof of (i) is to obtain $\lambda_{1}(\mu, \tau)<0$ by contradiction. Suppose that there exist $\tau_{i} \rightarrow 0^{+}$and $\mu_{i} \in\left(\underline{\mu}\left(\tau_{i}\right), \bar{\mu}\left(\tau_{i}\right)\right)$ such that $\lambda_{1}\left(\mu_{i}, \tau_{i}\right) \geq 0$ for $i=1,2, \ldots$ By passing to the limit, we have $\underline{\mu}\left(\tau_{i}\right) \rightarrow \mu_{1}, \bar{\mu}\left(\tau_{i}\right) \rightarrow \mu_{2}$ and $\mu_{i} \rightarrow \tilde{\mu}$ as $i \rightarrow \infty$ with $\tilde{\mu} \in\left[\mu_{1}, \mu_{2}\right]$.

For $\tilde{\mu} \in\left(\mu_{1}, \mu_{2}\right)$, we know that

$$
\lim _{i \rightarrow \infty} \frac{\lambda_{1}\left(\mu_{i}, \tau_{i}\right)}{\tau_{i}}=-\frac{G(\tilde{\mu}) H(\tilde{\mu})}{G(\tilde{\mu})+H(\tilde{\mu})} \frac{1}{\int_{\Omega} \theta^{2}(x, \tilde{\mu}) d x}<0
$$

from Lemma 4.2 and $G(\mu)>0, H(\mu)>0$ in $\left(\mu_{1}, \mu_{2}\right)$. Thus, for large $i$, we have $\lambda_{1}\left(\mu_{i}, \tau_{i}\right)<0$, which contradicts the assumption.

For $\tilde{\mu}=\mu_{1}$ or $\tilde{\mu}=\mu_{2}$, we still only consider the case $\tilde{\mu}=\mu_{1}$, and then the case $\tilde{\mu}=\mu_{2}$ can be treated similarly. In this case, we may suppose $G\left(\mu_{1}\right)=0$ (if $H\left(\mu_{1}\right)=0$, then it is analogous). It is clear that $G^{\prime}\left(\mu_{1}\right)>0$ since $G(\mu)>0$ in $\left(\mu_{1}, \mu_{2}\right)$ and $\mu_{1}$ is the simple root of $G(\mu)$. Therefore, we see

$$
\lim _{i \rightarrow \infty} \frac{\lambda_{1}\left(\mu_{i}, \tau_{i}\right)}{\tau_{i}\left(\mu_{i}-\underline{\mu}\left(\tau_{i}\right)\right)}=-\frac{G^{\prime}\left(\mu_{1}\right)}{\int_{\Omega} \theta^{2}\left(x, \mu_{1}\right) d x}<0,
$$

which also leads to $\lambda_{1}\left(\mu_{i}, \tau_{i}\right)<0$ for large $i$ combining with $\tau_{i} \rightarrow 0^{+}$and $\mu_{i} \in\left(\underline{\mu}\left(\tau_{i}\right), \bar{\mu}\left(\tau_{i}\right)\right)$. The contradiction completes the proof.

Remark 4.1 Theorem 4.1 is contrary to the stability results in [21]. But it is shown that the two competition species with different intraspecific competition rates can also coexist for bigger regions of $\mu$, which is similar to [21].

\section{Competing interests}

The authors declare that they have no competing interests.

\section{Authors' contributions}

MW performed the theory analysis and carried out the computations. JC participated in the design of the study and helped to draft the manuscript. LQ conceived of the study and participated in some computations. All authors have read and approved the final manuscript.

\section{Author details}

${ }^{1}$ Department of Mathematics and Statistics, Yulin University, Yulin, Shaanxi 719000, P.R. China. ${ }^{2}$ Institute of Information Engineering, Chinese Academy of Sciences, Beijing, 100093, P.R. China.

\section{Acknowledgements}

The authors would like to express sincere thanks to the anonymous referee for his/her carefully reading the manuscript and valuable comments on this paper. The work is supported by the Education Committee Foundation of Shaanxi Province, and the National Natural Science Foundation of China (No. 11271236).

Received: 12 February 2014 Accepted: 14 May 2014 Published: 30 May 2014

\section{References}

1. Bertness, MD: Intraspecific competition and facilitation in a northern acorn barnacle population. Ecology 70, 257-268 (1989)

2. Bürger, R: A multilocus analysis of intraspecific competition and stabilizing selection on a quantitative trait. J. Math. Biol. 50, 355-396 (2005)

3. Svanbäck, R, Bolnick, D: Intraspecific competition drives increased resource use diversity within a natural population. Proc. R. Soc. B 274, 839-844 (2007) 
4. Lankau, RA: Rapid evolutionary change and the coexistence of species. Annu. Rev. Ecol. Evol. Syst. 42, 335-354 (2011)

5. Hastings, A: Spatial heterogeneity and ecological models. Ecology 71, 426-428 (1990)

6. Takeuchi, Y: Global Dynamical Properties of Lotka-Volterra Systems. World Scientific, Singapore (1996)

7. Du, YH: Realization of prescribed patterns in the competition model. J. Differ. Equ. 193, 147-179 (2003)

8. Cantrell, RS, Cosner, C, Lou, Y: Multiple reversals of competitive dominance in ecological reserves via external habitat degradation. J. Dyn. Differ. Equ. 16, 973-1010 (2004)

9. Győri, I: Global attractivity in delay Lotka-Volterra differential equations. In: Fourth International Conference on Dynamic Systems and Applications, Atlanta, 21-24 May 2003, pp. 21-24 (2003)

10. Pao, CV: Global asymptotic stability of Lotka-Volterra competition systems with diffusion and time delays. Nonlinear Anal., Real World Appl. 5, 91-104 (2004)

11. Kuto, K, Yamada, Y: Positive solutions for Lotka-Volterra competition systems with large cross-diffusion. Appl. Anal. 89 1037-1066 (2010)

12. Hetzer, G, Nguyen, T, Shen, W: Coexistence and extinction in the Volterra-Lotka competition model with nonlocal dispersal. Commun. Pure Appl. Math. 11, 1699-1722 (2012)

13. Wu, DY: Global attractivity of a discrete Lotka-Volterra competition system with infinite delays and feedback controls. Adv. Differ. Equ. 2013, 14 (2013)

14. $\mathrm{He}, \mathrm{XQ}, \mathrm{Ni}, \mathrm{WM}$ : The effects of diffusion and spatial variation in Lotka-Volterra competition-diffusion system I: heterogeneity vs. homogeneity. J. Differ. Equ. 254, 528-546 (2013)

15. $\mathrm{He}, \mathrm{XQ}, \mathrm{Ni}, \mathrm{WM}$ : The effects of diffusion and spatial variation in Lotka-Volterra competition-diffusion system II: the general case. J. Differ. Equ. 254, 4088-4108 (2013)

16. Meng, XZ, Liu, R, Zhang, TH: Adaptive dynamics for a non-autonomous Lotka-Volterra model with size-selective disturbance. Nonlinear Anal., Real World Appl. 16, 202-213 (2014)

17. Dockery, J, Hutson, V, Mischaikow, K, Pernarowski, M: The evolution of slow dispersal rates: a reaction-diffusion model. J. Math. Biol. 37, 61-83 (1998)

18. Hutson, V, Mischaikow, K, Poláčik, P: The evolution of dispersal rates in a heterogeneous time-periodic environment. J. Math. Biol. 43, 501-533 (2001)

19. Hutson, V, Lou, Y, Mischaikow, K, Poláčik, P: Competing species near the degenerate limit. SIAM J. Math. Anal. 35 453-491 (2003)

20. Hetzer, G, Nguyen, $T$, Shen, W: Effects of small spatial variation of the reproduction rate in a two species competition model. Electron. J. Differ. Equ. 2010, 160 (2010)

21. Lou, Y, Martínez, S, Poláčik, P: Loops and branches of coexistence states in a Lotka-Volterra competition model. J. Differ. Equ. 230, 720-742 (2006)

22. Cantrell, RS, Cosner, C: Spatial Ecology via Reaction-Diffusion Equations. Wiley, Chichester (2003)

23. Deimling, K: Nonlinear Functional Analysis. Springer, Berlin (1985)

24. Hess, P: Periodic Parabolic Boundary Value Problems and Positivity. Longman, Harlow (1985)

25. Golubitsky, M, Schaeffer, D: Singularities and Groups in Bifurcation Theory, vol. I. Springer, New York (1985)

26. Kato, T: Perturbation Theory for Linear Operators. Springer, New York (1985)

10.1186/1687-1847-2014-159

Cite this article as: Wei et al.: Existence and stability of positive steady-state solutions for a Lotka-Volterra system with intraspecific competition. Advances in Difference Equations 2014, 2014:159

\section{Submit your manuscript to a SpringerOpen ${ }^{\circ}$ journal and benefit from:}

- Convenient online submission

Rigorous peer review

- Immediate publication on acceptance

- Open access: articles freely available online

- High visibility within the field

- Retaining the copyright to your article 